\title{
Evacuation Plans and Simulations for Crowd Egress - A Review
}

\author{
Purva Goyal, Dr. Akash Saxena
}

\author{
M.Tech Scholar, Computer Science and Engineering, CITM, Jaipur, India \\ purvagoyal27@gmail.com \\ Professor, Computer Science and Engineering, CITM, Jaipur, India \\ akash27saxena@gmail.com
}

\begin{abstract}
Due to growing population density and increasing complexity and congestion of human habitat evacuation planning is essential to avoid major loss oflife during a natural or un-natural disaster. The paper provides a review of existing evacuation systems and strategies and also points out the possible research directions. Intelligent Evacuation Management System coupled with evolutionary and machine learning techniques such as PSO,ACO, $k$-mean clustering is a promising solution to ensure safe and jostle free egress of people. .
\end{abstract}

Keywords-Evacuation system, Particle Swarm Optimization, K means clustering.

\section{INTRODUCTION}

THE advancement in science and technology as well as manifold increase in population density has increased the complexity of human habitat. Convolutedness and colossally are essential characteristics of modern day's buildings. Consequently the human life suffers from an imminent threat in case of natural disasters. As such situation forces human beings to take split seconds decisions. The number of causalities increases exponentially in lack of proper evacuation planning In 2014 a report was submitted by National Crime Records Bureau Of India[4] which stated that out of a total 20,201 deaths occurred due to natural caused and 6.36.509 deaths occurred due to un-natural causes.The "un-natural causes" are defined as accidents that result due to human negligencesuch as electrocution, traffic accidents, fire accidents etc. The statistics are alarming therefore government has declared evacuation planning as compuls ory.

The term evacuation can be defined as forced movement of people from a building or facility in case of an emergency. To ensure safe evacuation of people, the evacuation system must be planned in advance by considering the possible hazard scenarios. Standards are laid by government for designing an implementation plan such as ISO 23601:2009[3]. These standards suggests that proper exit signs and markings should be placed at visible places and exit routes must be communicated well in advance.

However due to widespread panic and jostle, evacuation of occupants is herculean task. Trained professionals should be present at the exit routes to guide the people. Additionally population classification is essential to ensure that no one is left behind. Walking speed of people differ with their age[1][2] as given in table 1.1.

In this paper the different evacuation systems are studied to identify literature gap and suggest possible research directions. The rest of the paper is organized as follows. Section II summarizes the related work in this domain. Section III summarizes existing evacuation systems. Research gap is listed in Section IV. Section V concludes the paper and Section VI mentions future research directions.

Table.1.1: [2] Different Walking Speeds of Human Beings

\begin{tabular}{|l|c|c|}
\hline \multicolumn{3}{|c|}{ Free Speed For Pedestrian Facilities $(\mathrm{m} / \mathbf{s})$} \\
\hline Male & Children & 0.72 \\
\cline { 2 - 3 } & Young & 0.79 \\
\cline { 2 - 3 } & Elder & 0.58 \\
\hline \multirow{4}{*}{ Female } & Children & 0.60 \\
\cline { 2 - 3 } & Young & 0.67 \\
\cline { 2 - 3 } & Elder & 0.52 \\
\hline
\end{tabular}

\section{RELATED WORK}

Yang and etal [5] proposed an intelligent fire evacuation system based on Ant Colony Optimization and MapX.The software calculated shortest path by fire points and ant colony optimization and updated lamp status on floor.Junaedi and etal[6] proposed an evacuation plan in case of fire break by employing multi agent behavior and Particle Swarm Optimization(PSO). The authors 
emphasized the importance of a leader to prevent jostling and panic in crowd. Turk and etal[7] tested the efficiency of PSO for network mitigation planning.and etal[8] proposed that stability of any system is affected by asymptotic growth rate of minimum evacuation time.Or in other words, stability of a system can be incurred by assessing optimum evacuation time policy. The proposed system was successfully applied to wireless networks. Xiangwei[9]proposed an algorithm for metro traffic route assignment by using K-means clustering algorithm and Class Conditional Probabilities. The database was created from Origin-Destination data of Beijing Metro Networks.Purnawansyah and Hailuddin[10] proposed a novel method for controlling the bandwidth usage in a university by developing a network clustering model based on K-means clustering algorithm. Bandwidth control is essential to support all learning and teaching activities. Yingqiu and etal[11] used K-means clustering to obtain an accurate and precise network traffic classification. Thus it can be affirmed that $\mathrm{K}$ mean clustering is an efficient classification algorithm and can be applied in evacuation planning for classifying evacuee population to ensure formation of proper groups and colossal free evacuation system.In addition to population classification, sorting algorithms must be employed in evacuation planning to ensure that group moves with an average speed so that no one is left behind. Yang and etal[12] compared the five sorting algorithms namely bubble sort, insertion sort, merger sort and quick sort. The results demonstrated superior performance of bubble sort in an ordered dataset. Tina-Yu and etal[13]proffered a shortest path evacuation algorithm in case of a fire evacuation system. Various sensors such as smoke and temperature sensors, RFID provide input to the algorithm in terms of smoke and population density. The central computer then calculates the shortest path and the path is displayed by intelligent indicator lamps. Lan[14] performed a comparative analysis of neural network based Ant Colony Optimization(ACO), Particle Swarm Optimization(PSO) and Genetic algorithm in terms of network traffic prediction. The results revealed that PSO is superior both in terms of accuracy and steady prediction as compared to other algorithms. Since network traffic prediction scenario is quite similar to an evacuation scenario, thus PSO should be applied in planning and developing an evacuation systems. Kulkarni and Agashe[15] performed an exhaustive review of existing fire evacuation systems. The authors also discussed various egress components, evacuation models and strategies, and different crowd monitoring techniques. In the paper Intelligent Evacuation Management System(IEMS) as amalgamation of crowd monitoring , disaster prediction and evacuation models.

\section{EXISTING EVACUATION SYSTEMS}

The literature reveals three types of existing evacuation systems as mentioned below.

3.1 Traditional Evacuation System: Exit marking and exit routes characterizes this type of evacuation system.

3.2 Optimization Based Evacuation Models : These system are Linear Programming based evacuation plans and works by calculating shortest path by utilizing computational algorithms such as Ant Colony Optimization [5] and Dijkstra Algorithm[13]. The calculated shortest path is displayed by intelligent sensor lamps [15] or floor topology maps [5].

3.3 Intelligent Evacuation Management System(IEMS):

Egress components and sensor information is used effectively along with evacuee information in Intelligent Evacuation Management Systems to make an agile and safe evacuation. This system can be characterized on the basis of occupant's movements as follows:

3.3.1 Coarse Approach Model : In this approach the infrastructure is divided into series of nodes and arcs. Despite being the simplest approach it is not popular as it does not considers human behavior.

3.3.2 Fine Approach Models : Individual's movement can be tracked in this model as the model divides the space into cells and each individual occupies a particular cell.

3.3.3 Continuous Approach Models : This is the most beneficial model, especially in case of high rise builidings as it not only tracks the location of individuals but also update it continuously.

\section{RESEARCH GAP}

Traditional evacuation systems have now become obsolete as they indicate only a fixed direction and may mislead people to a dangerous or wrong place. Optimization based evacuation models calculate shortest path by Dijkstra algorithim, simulated annealing, genetic algorithim etc. However this model doesnot takes into account the dynamics of real life situations and may lead to congestion. In the present scenario of complex facilities Intelligent evacuation Management System is a promising solution for easy, quick and hastle free evacuation. However only a few studies are dedicated towards this system. 


\section{CONCLUSION}

In the research work, it is noticed that in that modern day buildings are multifunctional and convolute. Catastropic disasters may occur, for instance fire or earthquake and result in widespread loss of life. However the number of casualities can be minimized by proper and in time evacuation planning. Intelligent Evacuation Management System is a prominent solution.

\section{FUTURE SCOPE}

Machine learning algorithms such as K-means clustering and evolutionary algorithms such as Particle Swarm Optimization and Ant Colony Optimization can serve as inspiration for developing an agile and safe evacuation system In future a real time evacuation system must be simulated based on the aforementioned technique.

\section{REFERENCES}

[1] M.I .Tolea, P.T .Costa, A .Terracciano, et al. "SexSpecific Correlates of Walking Speed in a Wide AgeRanged Population." The Journals of Gerontology Series B: Psychological Sciences and Social Sciences. 2010;65B(2):174-184. doi:10.1093/geronb/gbp130.

[2] S. Jiten, J. Gaurang, A. Shriniwas and P. Purnima, "Estimation of free speed of pedestrian flow on stairways at busy suburb rail transit station in India", current science, vol. 113, no. 5, pp 927-937 september 2017.

[3] "Accidental Deaths \& Suicides In India", A. Kumar, M. Rajakumar and S. Soni(Eds). National Crime Records Bureau Ministry of Home Affairs, Government of India, http://ncrb.gov.in. https://www.is o.org/obp/ui/\#iso:std:iso:23601:en.

[4] J. Yang, M. S., Z. Han, "Research intelligent fire evacuation system based on ant colony algorithm and MapX", Seventh International Symposium on Computational Intelligence and Design, IEEE, 2014.

[5] H. Junaedi , M. Hariadi, I. K.E.Purnama, "Multi Agent With Multi Behaviour Based on Particle Swarm Optimization (PSO) for Crowd Movement in Fire Evacuation Hartarto", Fourth International Conference on Intelligent Control and Information Processing (ICICIP),IEEE, 2013.

[6] S. Turk,X. Liu, R. Radeke, R. Lehnert, "Particle Swarm Optimization of Network Migration Planning",IEEE,2013.
[7] L.S Georgiadis, G. S. Paschos, L. Libman and L. Tassiulas, "Minimal Evacuation Times and Stability", IEEE, 2014.

[8] F. Xiangwei , L. Biao and X. Zhang, "Metro Traffic Route Assignment Using K-Means Clustering",IEEE,2011.

[9] Purnawansyah and Haviluddin, "K-Means Clustering Implementation in Network Traffic Activities", IEEE, 2016.

[10] L. Yingqiu, L. Wei and L. Yunchun, "Network Traffic Classification Using K-means Clustering", IEEE, 2007.

[11] Y. Yang, P. Yu and Y. Gan, "Experimental Study on the Five Sort Algorithms", IEEE ,2011.

[12] W. T.Yu ,H. Rui ,L. Lei ,X. Wen-guo and N. Ju-gen , "The Application of the Shortest Path Algorithm in the Evacuation System",IEEE,2011.

[13] XuLan, "Analysis and Research of several Network Traffic Prediction Models",IEEE,2013.

[14] Samruddhi S. Kulkarni and Prof. S. D. Agashe , "Study of Intelligent Evacuation Systems of HighRise Buildings in India-A Review",IEEE,2016. 